\title{
SCIENTIFIC REPORTS

\section{Differential protein expression of DARPP-32 versus Calcineurin in the prefrontal cortex and nucleus accumbens in schizophrenia and bipolar disorder}

\begin{abstract}
Yasuto Kunii ${ }^{1,2,7^{*}}$, Mizuki Hino ${ }^{1,7}$, Junya Matsumoto ${ }^{1}{ }^{1}$, Atsuko Nagaoka ${ }^{1}$, Hiroyuki Nawa ${ }^{3}$, Akiyoshi Kakita ${ }^{4}$, Hiroyasu Akatsu ${ }^{5,6}$, Yoshio Hashizume ${ }^{6}$ \& Hirooki Yabe ${ }^{1}$

Dopamine- and cAMP-regulated phosphoprotein of molecular weight $32 \mathrm{kDa}$ (DARPP-32) integrates dopaminergic signaling into that of several other neurotransmitters. Calcineurin (CaN), located downstream of dopaminergic pathways, inactivates DARPP-32 by dephosphorylation. Despite several studies have examined their expression levels of gene and protein in postmortem patients' brains, they rendered inconsistent results. In this study, protein expression levels of DARPP- 32 and $\mathrm{CaN}$ were measured by enzyme-linked immunosorbent assay (ELISA) in the prefrontal cortex (PFC), and nucleus accumbens (NAC) of 49 postmortem samples from subjects with schizophrenia, bipolar disorder, and normal controls. We also examined the association between this expression and genetic variants of 8 dopaminergic system-associated molecules for 55 SNPs in the same postmortem samples. In the PFC of patients with schizophrenia, levels of DARPP-32 were significantly decreased, while those of $\mathrm{CaN}$ tended to increase. In the NAc, both of DARPP-32 and CaN showed no significant alternations in patients with schizophrenia or bipolar disorder. Further analysis of the correlation of DARPP-32 and CaN expressions, we found that positive correlations in controls and schizophrenia in PFC, and schizophrenia in NAc. In PFC, the expression ratio of DARPP-32/CaN were significantly lower in schizophrenia than controls. We also found that several of the aforementioned SNPs may predict protein expression, one of which was confirmed in a second independent sample set. This differential expression of DARPP-32 and $\mathrm{CaN}$ may reflect potential molecular mechanisms underlying the pathogenesis of schizophrenia and bipolar disorder, or differences between these two major psychiatric diseases.
\end{abstract}

Dopamine- and cAMP-regulated phosphoprotein of molecular weight $32 \mathrm{kDa}$ (DARPP-32), also known as phosphoprotein phosphatase-1 regulatory subunit $1 \mathrm{~B}$ (PPP1R1B), is a key molecule that integrates dopaminergic signaling into that of several other neurotransmitters, such as a glutamate ${ }^{1}$. Phosphorylated DARPP-32 regulates the physiological activities of various proteins ${ }^{2}$. Calcineurin $(\mathrm{CaN})$ is located downstream of dopaminergic and glutamatergic signaling and inactivates DARPP-32 by dephosphorylation ${ }^{3}$. CaN has also been associated with synaptic plasticity ${ }^{4}$ and neural cell apoptosis ${ }^{5}$. It has been reported that the $\mathrm{CaN}$ inhibitor tacrolimus induces psychotic side effects such as hallucinations, anxiety, paranoid delusions, dissociative fugues, and manic-like psychosis $^{6-8}$. Therefore, elucidating the signaling pathway that involves DARPP-32 and CaN may be critical to understanding schizophrenia and other psychiatric disorders.

\footnotetext{
${ }^{1}$ Department of Neuropsychiatry, School of Medicine, Fukushima Medical University, 960-1295, Fukushima, Japan. ${ }^{2}$ Department of Psychiatry, Aizu Medical Center, Fukushima Medical University, 969-3492, Fukushima, Japan. ${ }^{3}$ Department of Molecular Neurobiology, Brain Research Institute, Niigata University, 951-8585, Niigata, Japan. ${ }^{4}$ Department of Pathology, Brain Research Institute, Niigata University, 951-8585, Niigata, Japan. ${ }^{5}$ Department of Community-based Medical Education/Department of Community-based Medicine, Nagoya City University Graduate School of Medical Science, 467-8601, Aichi, Japan. ${ }^{6}$ Choju Medical Institute, Fukushimura Hospital, 441-8124, Aichi, Japan. ${ }^{7}$ These authors contributed equally: Yasuto Kunii and Mizuki Hino. *email: kunii@fmu.ac.jp
} 


\begin{tabular}{|l|l|l|l|l|}
\hline & Schizophrenia & $\begin{array}{l}\text { Bipolar } \\
\text { Disorder }\end{array}$ & Control & Cont vs Sz (p value) \\
\hline First sample set & 21 & 6 & 22 & - \\
\hline Number of samples & $13 / 8$ & $3 / 3$ & $14 / 8$ & X$^{2}$-test $\mathrm{p}=0.907$ \\
\hline Sex (M/F) & 68.9 & 66.0 & 63.7 & Welch's $t$-test $\mathrm{p}=0.195$ \\
\hline Mean age at death (year) & 16.1 & 17.0 & 14.7 & Welch's $t$-test $\mathrm{p}=0.770$ \\
\hline Mean PMI (h) & 16.0 & - & - \\
\hline Mean CP-eq (mg/day) & 653.5 & - & - & \\
\hline Second sample set & Schizophrenia & Others & Control & \\
\hline \multicolumn{5}{|l|}{} \\
\hline Number of samples & 2 & 6 & 26 & \\
\hline Sex (M/F) & $1 / 1$ & $4 / 2$ & $12 / 14$ & \\
\hline Mean age at death (year) & 72 & 70.8 & 85.8 & \\
\hline Mean PMI (h) & 20.7 & 4.3 & 9.6 & \\
\hline Mean CP-eq (mg/day) & 1012.5 & - & - & \\
\hline
\end{tabular}

Table 1. Characteristics of postmortem brain samples. First sample set was used protein expression study in diagnostic groups as well as genotyping. Second sample set was used to confirm the association between rs1801028 and protein expression. PMI, postmortem interval; CP eq, chlorpromazine equivalent dose.

Several studies have examined the expression of DARPP-32 in the postmortem prefrontal cortex (PFC) of patients with schizophrenia or bipolar disorder ${ }^{9-14}$, but only a few focused on other brain regions ${ }^{12,13,15-17}$. Besides, scarce reports have addressed the protein or mRNA expression of $\mathrm{CaN}$ in postmortem brains of patients with schizophrenia ${ }^{18,19}$, and no studies have investigated this expression in patients with bipolar disorder. DARPP-32 and $\mathrm{CaN}$ are mostly expressed on dopamine receptor-positive neurons in various brain regions, especially in the caudate, putamen, and nucleus accumbens (NAc) ${ }^{20,21}$. In addition, NAc is an important contributor to the pathogenesis of schizophrenia ${ }^{22}$; it has been especially implicated in generating so-called positive symptoms, such as delusions or hallucinations, on account of its major role in regulating dopamine release and midbrain dopamine system $^{23}$. Nonetheless, the expression of DARPP-32 or CaN in the postmortem NAc of the patients with schizophrenia has not been investigated. Thus, we investigated the expression of DARPP-32 and CaN in NAc and PFC of the patients with schizophrenia.

Meanwhile, numerous studies have attempted, without success, to replicate previously reported genetic associations of multiple candidate genes with schizophrenia. Therefore, in recent years, most researchers have extensively investigated the functional correlations between schizophrenia risk alleles and intermediate phenotypes using neuroimaging, and cognitive and neurophysiologic indexes. Kleinman et al. (2011) proposed "genetic neuropathology," a novel strategy in which the expression of mRNA and proteins in postmortem brains is used as ultimate intermediate phenotypes. They hypothesized that insights into the mechanisms of psychiatric diseases and identification of novel therapeutic targets will emerge from the association of genetic variations with relevant molecular phenotypes in postmortem brains ${ }^{24}$. Actually, several recent postmortem studies ${ }^{25-28}$, including our own ${ }^{16,29}$, have adopted this strategy and provided unique insights into genetic and molecular mechanisms underlying schizophrenia.

Therefore, in this study, we investigated the expression levels of DARPP-32 and CaN proteins in PFC and NAc, regions that receive dopaminergic input and are considered to be affected in schizophrenia. This analysis was performed in 49 postmortem samples from subjects with schizophrenia, bipolar disorder, and control subjects (Table 1) using enzyme-linked immunosorbent assay (ELISA). Moreover, we analyzed the associations between this expression and genetic variants of dopaminergic signaling-associated molecules for 55 single nucleotide polymorphisms (SNPs) in a much larger set of postmortem samples to examine the potential involvement of genetic variants in the pathophysiology of schizophrenia and bipolar disorder.

\section{Results}

Expression of DARPP-32 and Calcineurin in the diagnostic groups. In the PFC, we found that the expression levels of DARPP-32 evinced a significant decrease in patients with schizophrenia relative to controls $(\mathrm{t}(40.14)=2.12, \mathrm{p}=0.040)$ (Fig. 1a) and that the expression of $\mathrm{CaN}$ tended to increase in patients with schizophrenia $(\mathrm{t}(40.72)=1.97, \mathrm{p}=0.056)$ as compared with controls; the mean of CaN expression in 6 patients with bipolar disorder was higher than that of controls (Fig. 2a). In the NAc, the expression levels of DARPP-32 and CaN showed no significant differences in schizophrenia patients relative to controls $(\mathrm{t}[23.14]=1.54, \mathrm{p}=0.138$; and $t[34.16]=1.56, p=0.127$; respectively; Figs $1 b$ and $2 b$ ); by contrast, each mean of two protein expressions in bipolar patients were higher than that of controls respectively (Figs $1 \mathrm{~b}$ and $2 \mathrm{~b}$ ).

We also assessed whether the expression level of either DARPP-32 or CaN in patients with schizophrenia was correlated with the daily dosage of antipsychotics that was prescribed in the 3 months immediately preceding each death. There were no significant effects of antipsychotic medication on the expression level of any of these proteins in any brain region examined (all $p$ values $>0.5$ ).

Further we assessed the correlation of expression levels of DARPP-32 and CaN in PFC and NAc (Fig. 3). Positive correlations were observed between the expression levels of DARPP-32 and CaN of controls in PFC $(\mathrm{r}=0.585, \mathrm{p}=0.004)$ (Fig. 3a), patients with schizophrenia in PFC $(\mathrm{r}=0.645, \mathrm{p}=0.002)$ (Fig. 3a) and NAc 
a

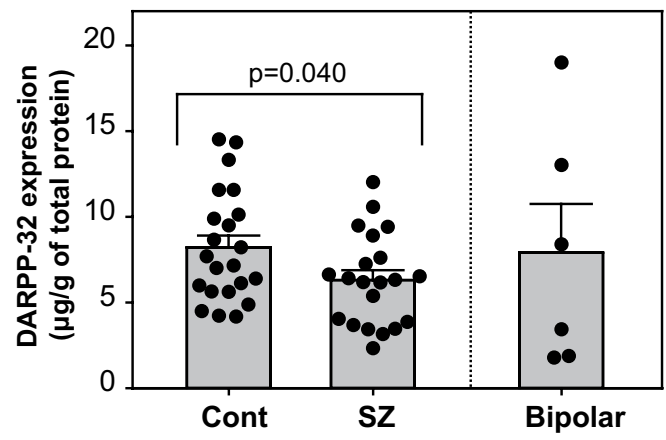

b

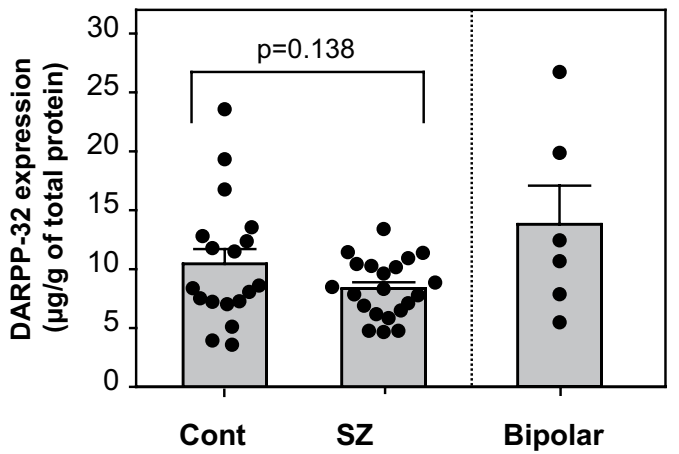

Figure 1. Expression of DARPP-32 in the diagnostic groups. DARPP-32 expression in the BA10 (a) and NAc (b) of the control group (Cont), the group of patients with bipolar disorder, and the group of patients with schizophrenia (SZ); ELISA was used to measure protein expression. Error bars indicate the standard error of the mean (SEM). p value between controls and schizophrenia was calculated using the Weich's t-test. Bipolar disorder was shown as a reference.

a

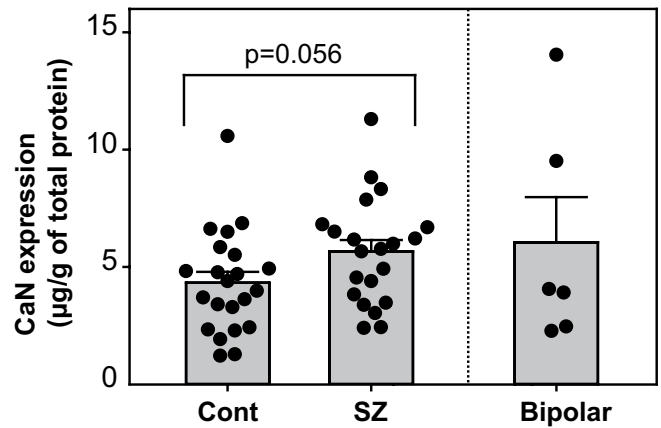

b

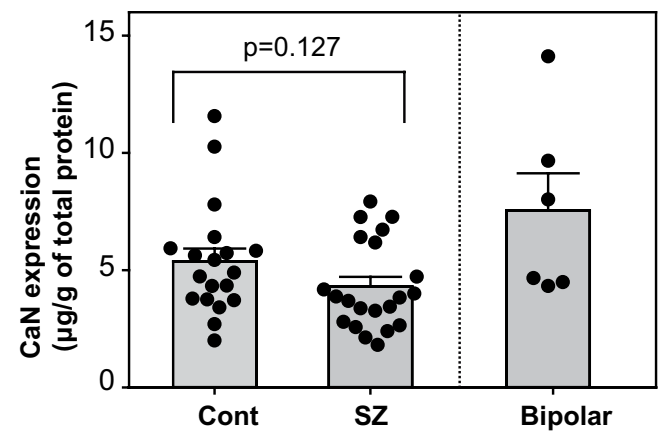

Figure 2. Expression of calcineurin in the diagnostic groups. Calcineurin $(\mathrm{CaN})$ expression in the BA10 (a) and NAc (b) of the control group (Cont), the group of patients with bipolar disorder, and the group of patients with schizophrenia (SZ); ELISA was used to measure protein expression. Error bars indicate the standard error of the mean (SEM). p value between controls and schizophrenia was calculated using Weich's t-test. Bipolar disorder was shown as a reference.

$(\mathrm{r}=0.550, \mathrm{p}=0.010)$ (Fig. $3 \mathrm{c})$. The ratio of these two proteins in each sample were compared between the diagnostic groups (Fig. 3b,d). In PFC, the ratio of DARPP-32/CaN in each sample were significantly low in schizophrenia patients compared to controls $(\mathrm{t}[29.72]=4.45, \mathrm{p}=1.12 \mathrm{e}-4)$ (Fig. 3b), but no changes were observed in NAc ( $\mathrm{t}[33.71]=-0.185, \mathrm{p}=0.854)$ (Fig. 3d).

Effects of dopaminergic system associated molecules genotype on expression of DARPP. 32 and calcineurin. We examined the effects of 55 SNPs in 8 dopaminergic system associated molecules (ANKK1, DRD2, PPP1R1B, PPP3CA, PPP3CB, PPP3CC, PPP3R1, and PPP3R2) on the protein expression levels of DARPP-32 or CaN in a cohort of patients and controls (Table 1) as described in Materials and Methods. The levels of protein expression were compared between minor allele carriers and non-carriers of these SNPs. The results are summarized in Table 2a. Significant associations were observed with 4 SNPs in the DRD2 (2), PPP3CB (1), and PPP3CC (1). These SNPs predicted protein expression (DARPP-32 or CaN) in the postmortem brains (BA10 or NAc). As shown in Table 2b, analysis of a second independent sample set confirmed only 1 SNPs out of 4SNPs: exm956405 (=rs1801028). There was a significant association between the expressions of CaN in the NAc and exm956405 (Fig. 4; first sample set: $U=10.0, p=0.016$; second sample set: $U=0.0, p=0.046$ ).

\section{Discussion}

Our study is the first analysis of DARPP-32 protein expression levels in the NAc of the patients with schizophrenia and those of $\mathrm{CaN}$ in the postmortem brain tissue of the patients with bipolar disorder. We showed that the levels of DARPP-32 in the PFC of the patients with schizophrenia significantly decreased, while those of CaN tended to increase in the PFC of the patients with schizophrenia. On the other hand, levels of DARPP-32 and CaN showed no significant differences in the NAc of the patients with schizophrenia or bipolar disorder. Moreover, in 
a

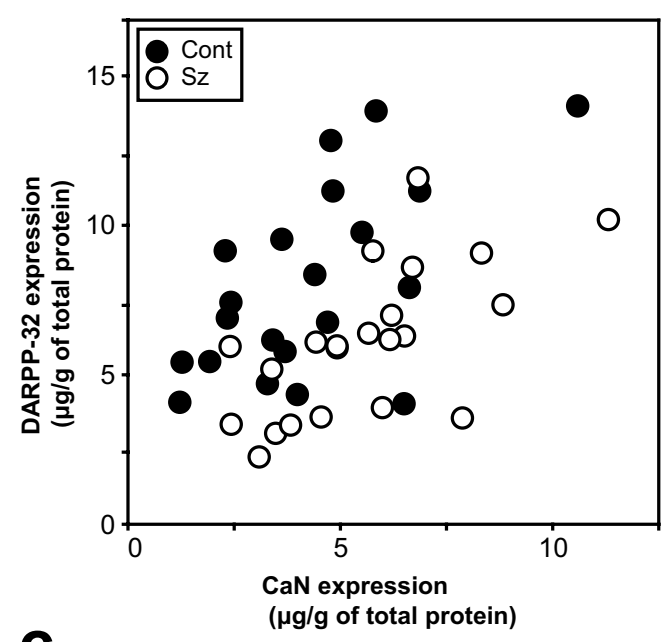

C

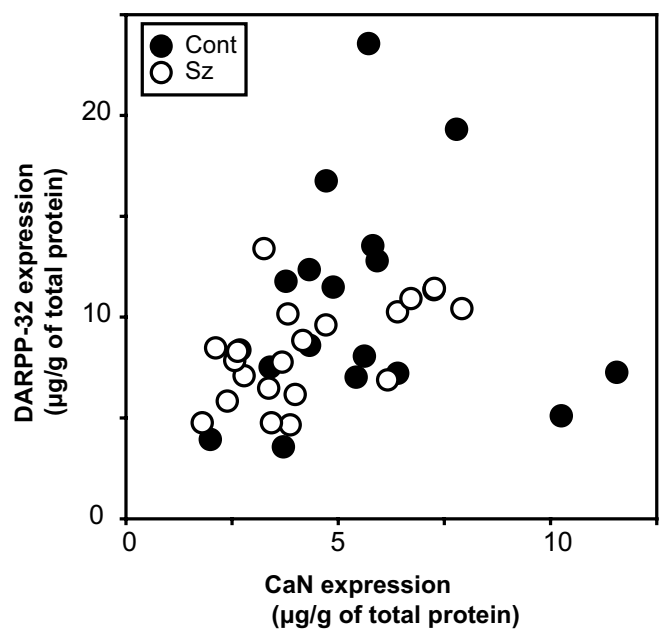

b
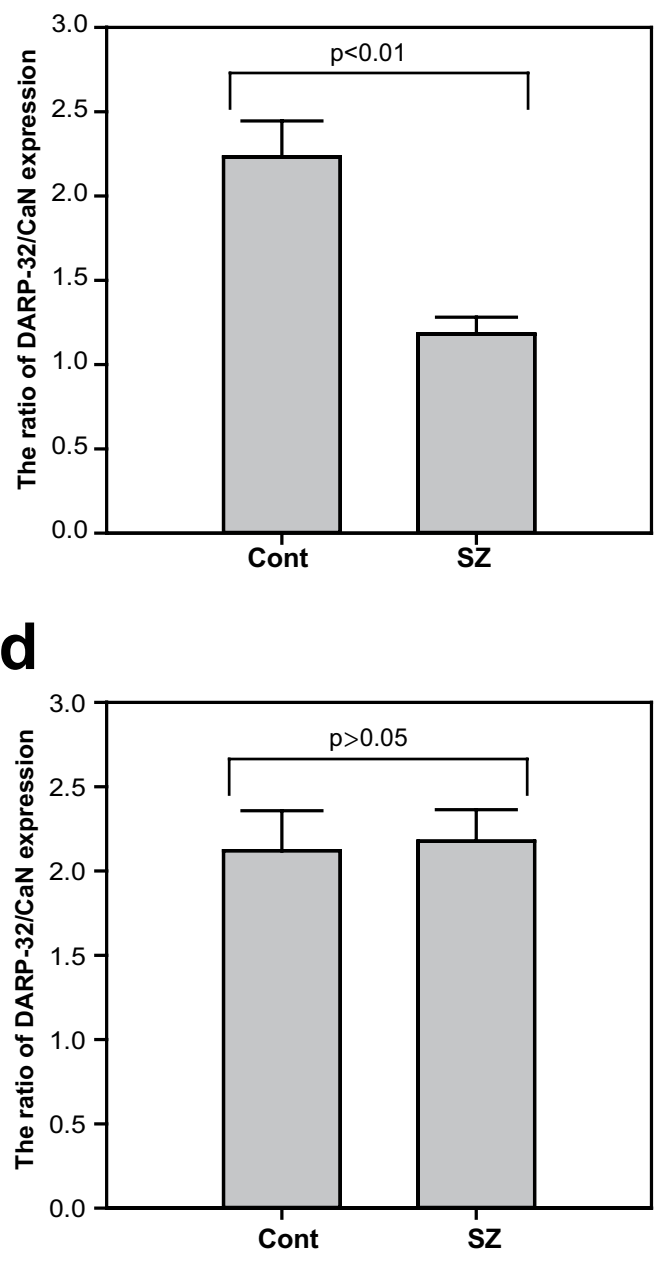

Figure 3. The correlation of the expression levels of DARPP-32 and CaN of control group and patients with schizophrenia. The expression levels of DARPP-32 and CaN in PFC (a) and NAc (c) of control group (closed circles) and patients with schizophrenia (open circles) are shown by scatter plots. The ratio of DARPP-32 and $\mathrm{CaN}$ expression of each sample were compared between diagnostic groups in PFC (b) and NAc (d). The $\mathrm{p}$ values between controls and schizophrenia were calculated using Welch's t-test. Error bars indicate SEM.

the PFC we found that positive correlations between expressions of DARPP-32 and CaN and the expression ratio of DARPP-32/CaN were significantly lower in schizophrenia than controls. As aforementioned, the expression of DARPP-32 in the postmortem brain of patients with schizophrenia or bipolar disorder has been largely investigated. While the levels of DARPP-32 protein in the PFC were decreased in patients with schizophrenia and bipolar disorder ${ }^{9,11,12}$, findings relevant to the mRNA levels in these regions were inconsistent across studies ${ }^{10,13,14,30,31}$. Alternatively, a few studies have investigated postmortem expression of $\mathrm{CaN}$ in the brains of the patients with schizophrenia ${ }^{18,19,32-34}$, but no studies have analyzed this expression in bipolar disorder. These postmortem studies of DARPP-32 or CaN in schizophrenia and/or bipolar disorder are summarized in Table 3. Anyway, the most interesting findings in this study was that in the PFC there were positive correlations between DARPP-32 and $\mathrm{CaN}$ expression, and lower DARPP-32/CaN ratio in schizophrenia, which were firstly reported to the best of our knowledge. We could point out several suggestions from these results: (1) DARPP-32 and CaN were usually coexistent in the brain, even if in schizophrenia, (2) the balance between DARPP-32 and CaN was greatly disrupted in schizophrenia, and (3) these ware consistent with the results of the above previous postmortem studies review.

If decreased DARPP-32/CaN expression ratio is the primary pathophysiological alteration in the patients with schizophrenia, that means that upregulation of CaN promotes the inactivation of DARPP- $32^{35}$. Decreased DARPP-32 activity induces a reduction in dopaminergic signaling in the PFC, which results in a so-called "hypofrontality" in schizophrenia. It is possible that a decrease in DARPP-32 expression in the PFC observed both in the current as well as in previous studies results, at least in part, from this process. On the contrary, it is of interest that the changes in CaN expression in the NAc evinced opposite tendencies in patients with schizophrenia and those with bipolar disorder. Since, upon the stimulation of dopamine $\mathrm{D}_{1}$ receptor, CaN inactivates DARPP-32 by 


\begin{tabular}{|c|c|c|c|c|c|c|c|c|c|}
\hline Gene & SNP & $\begin{array}{l}\text { exonic/ } \\
\text { intronic, and } \\
\text { substitution }\end{array}$ & Protein & Region & \begin{tabular}{|l|} 
The \\
numbers \\
of minor- \\
allele \\
carriers \\
and non- \\
carriers \\
\end{tabular} & $\begin{array}{l}\text { Hardy- } \\
\text { Weinberg } \\
\text { Equilibrium } \\
\text { ( } 2 \text { 2, P value) }\end{array}$ & \multicolumn{2}{|c|}{$\begin{array}{l}\text { The ratios } \\
\text { of protein } \\
\text { expression } \\
\text { between minor- } \\
\text { allele carriers } \\
\text { and non-carriers }\end{array}$} & $\begin{array}{l}\text { p value } \\
\text { (Mann- } \\
\text { Whitney) }\end{array}$ \\
\hline \multicolumn{10}{|c|}{ (a) First sample set } \\
\hline DRD2 & exm956405 (=rs1801028) & $\begin{array}{l}\text { exonic, Ser } \Rightarrow \\
\text { Cys }\end{array}$ & $\mathrm{CaN}$ & NAc & $3 / 42$ & $(10.2,0.001)$ & 0.55 & $(\mathrm{C}+/ \mathrm{C}-)$ & 0.016 \\
\hline$D R D 2$ & exm956405 (=rs1801028) & $\begin{array}{l}\text { exonic, Ser } \Rightarrow \\
\text { Cys }\end{array}$ & $\mathrm{CaN}$ & BA10 & $3 / 45$ & $(11.0,0.001)$ & 1.78 & $(\mathrm{C}+/ \mathrm{C}-)$ & 0.023 \\
\hline DRD2 & rs2734833 & intronic & DARPP & NAc & $3 / 41$ & $(44.0,0)$ & 0.67 & $(\mathrm{~T}+/ \mathrm{T}-)$ & 0.043 \\
\hline РРP3СB & exm2259632 (=rs 12644) & exonic & DARPP & BA10 & $34 / 14$ & $(1.92,0.166)$ & 1.35 & $(\mathrm{~T}+/ \mathrm{T}-)$ & 0.015 \\
\hline РРР3СC & rs1116084 & intronic & DARPP & BA10 & $37 / 11$ & $(0.756,0.385)$ & 1.68 & $(\mathrm{~A}+/ \mathrm{A}-)$ & 0.016 \\
\hline \multicolumn{10}{|c|}{ (b) Second sample set } \\
\hline Gene & SNP & $\begin{array}{l}\text { exonic/ } \\
\text { intronic, and } \\
\text { substitution }\end{array}$ & Protein & Region & & & \multicolumn{2}{|c|}{$\begin{array}{l}\text { The ratios } \\
\text { of protein } \\
\text { expression } \\
\text { between minor- } \\
\text { allele carriers } \\
\text { and non-carriers }\end{array}$} & $\begin{array}{l}\text { p value } \\
\text { (Mann- } \\
\text { Whitney) }\end{array}$ \\
\hline$D R D 2$ & exm956405 (=rs1801028) & $\begin{array}{l}\text { exonic, Ser } \Rightarrow \\
\text { Cys }\end{array}$ & $\mathrm{CaN}$ & NAc & $2 / 6$ & $(8,0.0047)$ & 0.37 & $(\mathrm{C}+/ \mathrm{C}-)$ & 0.046 \\
\hline
\end{tabular}

Table 2. Summary of allelic effects of SNPs of dopaminergic-system associated molecules on DARPP-32 and calcineurin expression.

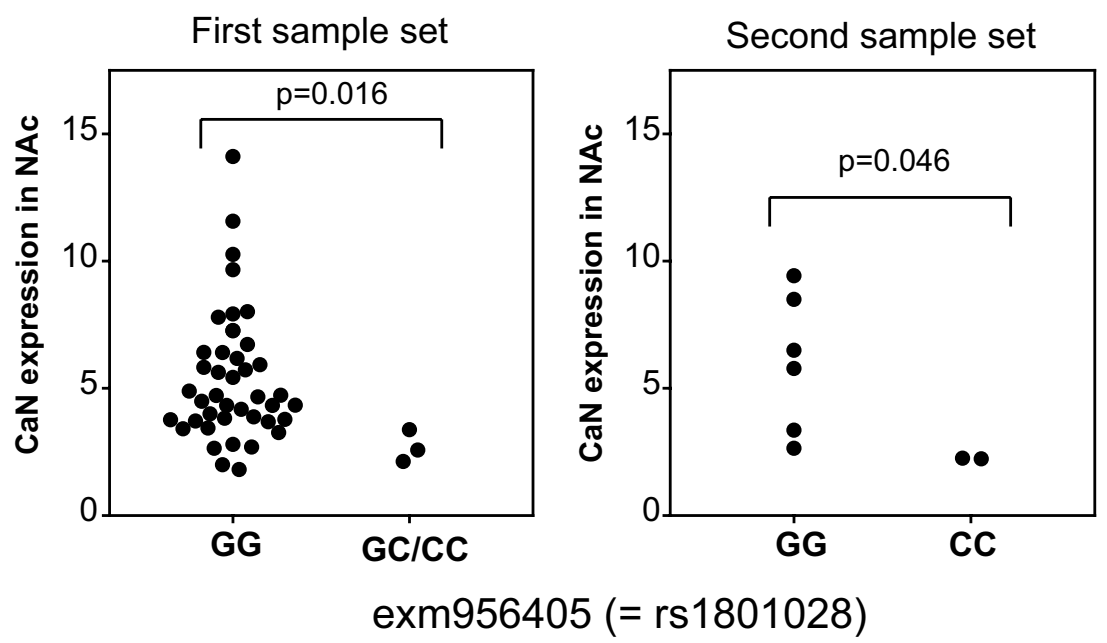

Figure 4. The allelic effect of exm956405 (=rs1801028) on the expression of calcineurin. A significant association was detected between the expression of CaN in the NAc and exm956405. The $p$-value between these groups was calculated using the Mann-Whitney U-test.

dephosphorylation, it can be concluded that $\mathrm{CaN}$ exerts a buffering action against excessive dopamine signaling. It is probable that variations in $\mathrm{CaN}$ expression may reflect different dopaminergic pathologies between these two major psychiatric diseases.

We also found that several SNPs of the gene encoding DRD2 and CaN were associated with DARPP-32 or $\mathrm{CaN}$ protein expression in postmortem brains. In particular, the polymorphism of protein phosphatase 3 catalytic subunit gamma (PPP3CC), a gene encoding the $\mathrm{CaN} \gamma$-catalytic subunit, was previously found to be relevant to schizophrenia. Genetic studies revealed a significant association between genetic variations of $P P P 3 C C^{36}$ or $P P P 3 C C$ gene haplotype ${ }^{37}$ and the incidence of schizophrenia; however, no correlations were found for other population samples ${ }^{38-40}$. In addition, an association between the PPP3CC gene and bipolar disorder was also reported $^{41}$. Another study using forebrain-specific PPP3CC gene-knockout mice reported multiple abnormal behaviors associated with schizophrenia, such as decreased social interaction and impairments in prepulse inhibition ${ }^{42}$.

In a second independent sample set, we could confirm only 1 SNP, exm956405 (=rs1801028), that predicted $\mathrm{CaN}$ expression in the NAc. This SNP was identified as a novel missense nucleotide change causing an amino acid substitution of serine with cysteine at codon 311 (Ser311Cys) in DRD2 ${ }^{43}$. An association study showed that Cys311 in DRD2 was significantly associated with schizophrenia ${ }^{44}$. Moreover, clinical assessment found that 


\begin{tabular}{|c|c|c|c|c|c|}
\hline Number of resources & Brain Region & Taget molecule & Method & Main findings & Refference \\
\hline \multicolumn{6}{|c|}{ (a) Postmortem brain studies of DARPP-32 } \\
\hline $\mathrm{SZ}(14), \mathrm{CONT}(14)$ & PFC & Protein & Immunoblot & DARPP-32 expression was reduced in SZ & Albert et al. ${ }^{9}$ \\
\hline $\mathrm{SZ}(12), \mathrm{BP}(10), \mathrm{CONT}(11)$ & PFC & Protein & IHC, Immunoblot & DARPP-32 expression was lower in SZ and BP & Ishikawa et al. ${ }^{11}$ \\
\hline $\operatorname{SZ}(9), \operatorname{CONT}(9)$ & $\begin{array}{l}\text { PFC, Hip, } \\
\text { Striatum }\end{array}$ & Protein & IHC & $\begin{array}{l}\text { DARPP-32 immnoreactive neurons were lower in } \\
\text { layers II-V of PFC in SZ }\end{array}$ & Kunii et al. ${ }^{12}$ \\
\hline $\operatorname{SZ}(11), \operatorname{CONT}(10)$ & Insula cortex & Protein & IHC & $\begin{array}{l}\text { DARPP-32 immnoreactive neurons were lower in } \\
\text { layers II-III of inslula in SZ }\end{array}$ & Nishiura et al..$^{17}$ \\
\hline $\mathrm{SZ}(11), \operatorname{CONT}(11)$ & STG & Protein & IHC & $\begin{array}{l}\text { DARPP-32 immnoreactive neurons were lower in } \\
\text { layers III-IV of STG in SZ }\end{array}$ & Kunii et al..$^{15}$ \\
\hline $\mathrm{SZ}(12), \mathrm{CONT}(12)$ & Striatum & Protein & Immunoblot & DARPP-32 expression was increased in CAU of SZ & Kunii et al..$^{16}$ \\
\hline $\mathrm{SZ}(13), \operatorname{CONT}(8)$ & Thalamus & mRNA & ISH & $\begin{array}{l}\text { No chnages in DARPP- } 32 \text { mRNA expression in } \\
\text { thalamic nuclei of SZ }\end{array}$ & Clinton et al..$^{31}$ \\
\hline $\operatorname{SZ}(18), \operatorname{CONT}(11)$ & PFC, ACC & mRNA & ISH & $\begin{array}{l}\text { No chnages in DARPP- } 32 \text { mRNA expression in } \\
\text { PFC and ACC of SZ }\end{array}$ & Baracskay et al..$^{10}$ \\
\hline $\mathrm{SZ}(35), \mathrm{BP}(35), \mathrm{CONT}(35)$ & PFC & mRNA & Realtime PCR & $\begin{array}{l}\text { DARPP- } 32 \text { mRNA expression was reduced in PFC } \\
\text { of SZ who died by suicide }\end{array}$ & Feldcamp et al. ${ }^{30}$ \\
\hline $\mathrm{SZ}(33), \mathrm{BP}(32), \mathrm{CONT}(34)$ & PFC & mRNA & Realtime PCR & $\begin{array}{l}\text { DARPP- } 32 \mathrm{mRNA} \text { expression was increased in PFC } \\
\text { of SZ and BP }\end{array}$ & Zhan et al. ${ }^{14}$ \\
\hline $\begin{array}{l}\text { SZ }(n=176), B P(n=61) \\
\text { MDD(138)CONT } \\
(n=283)\end{array}$ & PFC, Hip, Cau & mRNA & Realtime PCR & $\begin{array}{l}\text { t-DARPP mRNA expression was increased in PFC } \\
\text { of SZ and BP }\end{array}$ & Kunii et al. ${ }^{13}$ \\
\hline \multicolumn{6}{|c|}{ (b) Postmortem brain studies of $\mathrm{CaN}$} \\
\hline Number of resources & Brain Region & Taget molecule & Method & Main findings & Refference \\
\hline SZ(15), CONT(15) & PFC, Hip & Protein & Immunoblot & No chnages in CaN in PFC and Hip of SZ & Kozlovsky et al..$^{19}$ \\
\hline $\mathrm{SZ}(9), \operatorname{CONT}(9)$ & $\begin{array}{l}\text { PFC, Hip, } \\
\text { Striatum }\end{array}$ & Protein & IHC & $\begin{array}{l}\text { CaN immnoreactive neurons were increased in } \\
\text { caudate of SZ }\end{array}$ & Wada et al. ${ }^{33}$ \\
\hline $\mathrm{SZ}(12), \mathrm{CONT}(12)$ & Striatum & Protein & Immunoblot & No chnages in CaN in caudate and putamen of SZ & Kunii et al. ${ }^{16}$ \\
\hline SZ(11), CONT(11) & STG & Protein & IHC & $\begin{array}{l}\text { CaN immnoreactive neurons were increased in } \\
\text { layers II-VI of STG in SZ }\end{array}$ & Wada et al. ${ }^{34}$ \\
\hline $\mathrm{SZ}(12), \operatorname{CONT}(12)$ & PFC & mRNA & $\begin{array}{l}\text { DNA microarray } \\
\text { (cDNA) }\end{array}$ & CaN A mRNA was increased in PFC of SZ & Hakak et al..$^{32}$ \\
\hline SZ(13), CONT(12) & Hip & Protein, mRNA & ELISA, RT-PCR & CaN A and mRNA were decreased in hip of SZ & Eastwood et al..$^{18}$ \\
\hline
\end{tabular}

Table 3. Overview of postmortem brain studies focused on DARPP-32(a) and CaN(b). SZ, schizophrenia; CONT, control; BP, bipolar; PFC, prefrontal cortex; Hip, hippocampus; STG, superior temporal gyrus; ACC, anterior cingulate gyrus; Cau, caudate; IHC, immunohistochemistry; ISH, in situ hybridization; ELISA, enzyme-linked immunosorbent assay; CaN, calcineurin.

patients with schizophrenia that exhibited the Cys311 allele spent less time sin the hospital, presented less severe negative symptoms, and were more often married compared to patients without Cys $311^{44}$. These results suggest that patients with schizophrenia that have the Cys311 allele may respond well to antipsychotic treatment and, as a result, shows relatively mild negative symptoms. A several meta-analyses also demonstrated this association ${ }^{45-47}$. The tendency of this association was observed in our samples of this study (relative risk; 2.968, Fisher's exact test; $\mathrm{p}=0.071$ ). It was further demonstrated that dopamine-induced internalization of DRD2 was impaired in the Cys311 variant of DRD2 ${ }^{48}$. Since the internalization of DRD2 contributes to its desensitization ${ }^{49}$, the desensitization of DRD2 may also be suppressed in patients with the Cys311 variant. Thus, the disturbance of DRD2 desensitization can prolong dopamine-induced DRD2-mediated signaling and accelerate the subsequent processes (Fig. 5). Eventually, dephosphorylation of cAMP response element-binding protein (CREB) binds upstream of four CaN genes (PPP3CA, PPP3CB, PPP3CC, PPP3R1; found through the ChIP-Atlas database at "chip-atlas. org") and then decreases CaN expression. This assumed mechanism (Fig. 5) was consistent with our finding that subjects with Cys311 in DRD2 featured lower levels of CaN in the NAc (Fig. 4). In other words, the C allele of rs1801028 was at risk for schizophrenia and was associated with lower CaN expression in the NAc.

The present study performed on brain samples has several limitations that require further attention. First, confounding effects of disease-related factors including medication may have affected the expression of DARPP-32, $\mathrm{CaN}$, or both. Although we did not observe any effects of antipsychotic drugs on the levels of DARPP-32 or CaN expression in this study, additional animal studies are necessary to investigate the effects of chronic antipsychotic administration on the expression of these proteins in the PFC and NAc. Second, our study population was relatively small, especially for a genetic study. In addition, we could not uniformly sample from group with sufficient equilibrium because the NAc was a relatively small area and there were a small number of NAc available. As a result, several genotypes in this study including rs1801028 were not two in Hardy-Weinberg Equilibrium. The findings must be, therefore, confirmed via postmortem examination of a larger brain cohort.

In conclusion, our results show that the expressions of the DARPP- 32 and CaN protein were altered in postmortem brains of the patients with schizophrenia and bipolar disorder compared to those from control subjects, which is accompanied by genetic variations. Our results may reflect potential molecular mechanisms underlying the pathogenesis of schizophrenia and bipolar disorder or differences between these two major psychiatric diseases. 


\author{
Impaired DA-induced internalization of DRD2 \\ 1 \\ Increased DA-induced DRD2-mediated signaling \\ 1 \\ CaN activation by elevated intracellular $\mathrm{Ca}^{2+}$ level \\ L \\ PP1 activation through the dephosphorylation of DARPP-32 \\ Dephosphorylation of CREB \\ Decreased CaN expression
}

Figure 5. Flow chart of the DARPP-32-CaN pathway in the Cys 311 variant of DRD2. The disturbance of DRD2 desensitization can prolong dopamine-induced DRD2-mediated signaling and accelerate the following processes; PLC activation, IP3 release, and $\mathrm{Ca}^{2+}$ release from the endoplasmic reticulum. As a result, increased intracellular $\mathrm{Ca}^{2+}$ activates $\mathrm{CaN}$. The activated CaN can then promote PP1 activation through the dephosphorylation of DARPP-32. Dephosphorylation of CREB may then be promoted in the brains carrying the Cys311 variant of DRD2. Since the ChIP analysis showed that CREB binds upstream of several CaN genes, dephosphorylation of CREB may decrease CaN expression. DRD2, dopamine D2 receptor; PLC, phospholipase C; IP3, Inositol trisphosphate; CaN, calcineurin;PPI,;CREB, cAMP response element-binding protein; ChIP, Chromatin immunoprecipitation.

\begin{abstract}
Materials and Methods
Human postmortem brain tissue collection. Postmortem brain tissue samples from patients with schizophrenia, bipolar disorder, and control subjects were obtained from Fukushima Brain Bank at the Department of Neuropsychiatry, Fukushima Medical University; Brain Research Institute, Niigata University; and Choju Medical Institute Fukushimura Hospital, Toyohashi as described previously ${ }^{29}$. Use of postmortem human brain tissues for the present study was approved by the Ethics Committee of Fukushima Medical University and Niigata University, and complied with the Declaration of Helsinki and its later amendments. All procedures were carried out with the informed written consent of the next of kin. Detailed demographic information of brain tissues from 21 subjects with schizophrenia, 6 subjects with bipolar disorder and 22 control subjects used in this study (it was identical to first sample set in genotyping) was summarized in Table 1. Each patient with schizophrenia and bipolar disorder fulfilled the diagnostic criteria established by the American Psychiatric Association (Diagnostic and Statistical Manual of Mental Disorders: DSM-IV). For patients with schizophrenia, the daily dosage of antipsychotics prescribed during the 3 months immediately preceding death is shown as chlorpromazine-equivalent dose (mg/day, Table 1).
\end{abstract}

DNA collection and SNP genotyping. The subjects for the association analysis between SNPs and protein expression consisted of 27 patients (16 men; mean age, $68.2 \pm 15.2$ years) and 22 control subjects (14 men; mean age, $63.7 \pm 14.4$ years). A second replication sample set consisted of 34 subjects ( 17 men; mean age, $82.4 \pm 9.4$ years). Genomic DNA was extracted from the frozen cerebellum or occipital cortex and genotyping was performed using HumanCoreExome -24 v1.0 Beadchip on an iScan system (Illumina, Tokyo, Japan) as described previously ${ }^{29}$. Of 128 SNPs derived from 8 dopaminergic system-associated genes [Ankyrin repeat and kinase domain containing 1(ANKK1), dopamine D2 receptor (DRD2), phosphoprotein phosphatase-1 regulatory subunit 1B(PPP1R1B), protein phosphatase 3 catalytic subunit alpha (PPP3CA), protein phosphatase 3 catalytic subunit beta (PPP3CB), protein phosphatase 3 catalytic subunit gamma (PPP3CC), protein phosphatase 3 regulatory subunit B alpha (PPP3R1), and protein phosphatase 3 regulatory subunit B beta (PPP3R2)] included in the chip, we excluded SNPs with call rates of $<95 \%$ and minor allele frequencies of $<1 \%$; the number of SNPs analyzed thus totaled to 55 (Supplementary Table S1).

Tissue retrieval and processing for ELISA. Pieces (weighing approximately $100 \mathrm{mg}$ ) of gray matter tissue from Brodmann area 10 (BA10) as PFC and NAc were isolated from frozen brain. For PFC, gray matter tissue was dissected from the frontal pole. NAc was identified on the frozen coronal slabs corresponding to the anterior one-third of the inferior temporal cortex. These frozen brain tissues were suspended in the $2 \%$ SDS solution, incubated for $20 \mathrm{~min}$, subjected to three freeze-thaw cycles, and sonicated for $10 \mathrm{~min}$. Then, the samples were diluted in phosphate buffered saline (PBS; $137 \mathrm{mM} \mathrm{NaCl}, 2.7 \mathrm{mM} \mathrm{KCl}, 10 \mathrm{mM} \mathrm{Na}_{2} \mathrm{HPO}_{4}, 1.76 \mathrm{mM} \mathrm{KH}_{2} \mathrm{PO}_{4}$ ) so that the final concentration of SDS was below $0.2 \%$. The expression of proteins was determined by using commercial ELISA kits (SEB323Hu for Calcineurin, and SEE017Hu for DARPP-32, Cloud Clone Corp, Houston, TX). The analysis was performed according to the manufacturer's protocols. The expression levels of each protein were normalized against the total protein concentration.

Statistical analysis. Demographic variables (sex, age, and PMI) were compared between the groups using a $\chi^{2}$-test and Welch's t-test. The latter was also used for comparing the levels of protein (DARPP-32, $\mathrm{CaN}$ ) expression between the diagnostic groups (schizophrenia and control, with bipolar disorder as a reference). The correlations of expression levels of DARPP-32 and CaN in each sample were assessed by Pearson's correlation coefficients, and the ratio of proteins were compared between the diagnostic groups by Welch's t-test. For association study of SNPs, we divided all samples into minor allele carriers and non-carriers of each SNPs. 
A Mann-Whitney $U$-test was then used for comparing the levels of protein expression between the genotypes of each SNP. In the comparison between the two groups, we excluded outliers ( $>$ mean $\pm 3 \mathrm{SD})$ from analysis. Further, Spearman's rank correlation coefficients were used to assess the association between the expressions of each protein and estimated total dosage of neuroleptic drugs. SPSS ver. 25.0 (SPSS, Chicago, IL, USA) and (SigmaPlot14.0; Systat Software Inc., San Jose, CA, USA) was used for analysis.

Received: 17 July 2019; Accepted: 1 October 2019;

Published online: 16 October 2019

\section{References}

1. Lugo, J. N. et al. Deletion of PTEN produces autism-like behavioral deficits and alterations in synaptic proteins. Front Mol Neurosci 7, 27, https://doi.org/10.3389/fnmol.2014.00027 (2014).

2. Zhang, X., Liu, X., Wang, D., Liu, H. \& Hao, W. Conditioned place preference associated with level of palmitoylation of PSD-95 in rat hippocampus and nucleus accumbens. Neuropsychobiology 64, 211-218, https://doi.org/10.1159/000327603 (2011).

3. Nishi, A., Snyder, G. L., Nairn, A. C. \& Greengard, P. Role of calcineurin and protein phosphatase-2A in the regulation of DARPP-32 dephosphorylation in neostriatal neurons. J Neurochem 72, 2015-2021 (1999).

4. Baumgartel, K. \& Mansuy, I. M. Neural functions of calcineurin in synaptic plasticity and memory. Learn Mem 19, 375-384, https:// doi.org/10.1101/lm.027201.112 (2012).

5. Asai, A. et al. High level calcineurin activity predisposes neuronal cells to apoptosis. J Biol Chem 274, 34450-34458 (1999).

6. Bersani, G. et al. Manic-like psychosis associated with elevated trough tacrolimus blood concentrations 17 years after kidney transplant. Case Rep Psychiatry 2013, 926395, https://doi.org/10.1155/2013/926395 (2013).

7. Corruble, E. et al. Psychosis associated with elevated trough tacrolimus blood concentrations after combined kidney-pancreas transplant. Int J Neuropsychopharmacol 9, 493-494, https://doi.org/10.1017/S1461145705006279 (2006).

8. Krishna, N., Chiappelli, J., Fischer, B. A. \& Knight, S. Tacrolimus-induced paranoid delusions and fugue-like state. Gen Hosp Psychiatry 35, 327 e325-327 e326, https://doi.org/10.1016/j.genhosppsych.2012.07.010 (2013).

9. Albert, K. A. et al. Evidence for decreased DARPP-32 in the prefrontal cortex of patients with schizophrenia. Arch Gen Psychiatry 59, 705-712 (2002)

10. Baracskay, K. L., Haroutunian, V. \& Meador-Woodruff, J. H. Dopamine receptor signaling molecules are altered in elderly schizophrenic cortex. Synapse 60, 271-279, https://doi.org/10.1002/syn.20292 (2006).

11. Ishikawa, M., Mizukami, K., Iwakiri, M. \& Asada, T. Immunohistochemical and immunoblot analysis of Dopamine and cyclic AMPregulated phosphoprotein, relative molecular mass 32,000 (DARPP-32) in the prefrontal cortex of subjects with schizophrenia and bipolar disorder. Prog Neuropsychopharmacol Biol Psychiatry 31, 1177-1181, https://doi.org/10.1016/j.pnpbp.2007.04.013 (2007).

12. Kunii, Y. et al. Detailed DARPP-32 expression profiles in postmortem brains from patients with schizophrenia: an immunohistochemical study. Med Mol Morphol 44, 190-199, https://doi.org/10.1007/s00795-010-0524-1 (2011).

13. Kunii, Y. et al. Revisiting DARPP-32 in postmortem human brain: changes in schizophrenia and bipolar disorder and genetic associations with t-DARPP-32 expression. Mol Psychiatry 19, 192-199, https://doi.org/10.1038/mp.2012.174 (2014).

14. Zhan, L. et al. Altered expression and coregulation of dopamine signalling genes in schizophrenia and bipolar disorder. Neuropathol Appl Neurobiol 37, 206-219, https://doi.org/10.1111/j.1365-2990.2010.01128.x (2011)

15. Kunii, Y. et al. Altered DARPP-32 expression in the superior temporal gyrus in schizophrenia. Prog Neuropsychopharmacol Biol Psychiatry 35, 1139-1143, https://doi.org/10.1016/j.pnpbp.2011.03.016 (2011).

16. Kunii, Y. et al. Elevated postmortem striatal t-DARPP expression in schizophrenia and associations with DRD2/ANKK1 polymorphism. Prog Neuropsychopharmacol Biol Psychiatry 53, 123-128, https://doi.org/10.1016/j.pnpbp.2014.03.014 (2014).

17. Nishiura, K. et al. Profiles of DARPP-32 in the insular cortex with schizophrenia: a postmortem brain study. Prog Neuropsychopharmacol Biol Psychiatry 35, 1901-1907, https://doi.org/10.1016/j.pnpbp.2011.07.010 (2011).

18. Eastwood, S. L., Burnet, P. W. \& Harrison, P. J. Decreased hippocampal expression of the susceptibility gene PPP3CC and other calcineurin subunits in schizophrenia. Biol Psychiatry 57, 702-710, https://doi.org/10.1016/j.biopsych.2004.12.029 (2005).

19. Kozlovsky, N., Scarr, E., Dean, B. \& Agam, G. Postmortem brain calcineurin protein levels in schizophrenia patients are not different from controls. Schizophr Res 83, 173-177, https://doi.org/10.1016/j.schres.2005.12.843 (2006).

20. Ouimet, C. C., Miller, P. E., Hemmings, H. C. Jr. \& Walaas, S. I. \& Greengard, P. DARPP-32, a dopamine- and adenosine $3^{\prime}: 5^{\prime}$-monophosphate-regulated phosphoprotein enriched in dopamine-innervated brain regions. III. Immunocytochemical localization. J Neurosci 4, 111-124 (1984).

21. Ouimet, C. C., LaMantia, A. S., Goldman-Rakic, P., Rakic, P. \& Greengard, P. Immunocytochemical localization of DARPP-32, a dopamine and cyclic-AMP-regulated phosphoprotein, in the primate brain. J Comp Neurol 323, 209-218, https://doi.org/10.1002/ cne.903230206 (1992).

22. Mikell, C. B. et al. The hippocampus and nucleus accumbens as potential therapeutic targets for neurosurgical intervention in schizophrenia. Stereotact Funct Neurosurg 87, 256-265, https://doi.org/10.1159/000225979 (2009).

23. Haber, S. N., Fudge, J. L. \& McFarland, N. R. Striatonigrostriatal pathways in primates form an ascending spiral from the shell to the dorsolateral striatum. J Neurosci 20, 2369-2382 (2000).

24. Kleinman, J. E. et al. Genetic neuropathology of schizophrenia: new approaches to an old question and new uses for postmortem human brains. Biol Psychiatry 69, 140-145, https://doi.org/10.1016/j.biopsych.2010.10.032 (2011).

25. Dickinson, D. et al. Differential effects of common variants in SCN2A on general cognitive ability, brain physiology, and messenger RNA expression in schizophrenia cases and control individuals. JAMA Psychiatry 71, 647-656, https://doi.org/10.1001/ jamapsychiatry.2014.157 (2014).

26. Morita, Y. et al. Characteristics of the cation cotransporter NKCC1 in human brain: alternate transcripts, expression in development, and potential relationships to brain function and schizophrenia. J Neurosci 34, 4929-4940, https://doi.org/10.1523/ JNEUROSCI.1423-13.2014 (2014).

27. O'Donovan, S. M. et al. Glutamate transporter splice variant expression in an enriched pyramidal cell population in schizophrenia. Transl Psychiatry 5, e579, https://doi.org/10.1038/tp.2015.74 (2015).

28. Ohi, K. et al. DEGS2 polymorphism associated with cognition in schizophrenia is associated with gene expression in brain. Transl Psychiatry 5, e550, https://doi.org/10.1038/tp.2015.45 (2015).

29. Hino, M. et al. Decreased VEGFR2 expression and increased phosphorylated Akt1 in the prefrontal cortex of individuals with schizophrenia. J Psychiatr Res 82, 100-108, https://doi.org/10.1016/j.jpsychires.2016.07.018 (2016)

30. Feldcamp, L. A., Souza, R. P., Romano-Silva, M., Kennedy, J. L. \& Wong, A. H. Reduced prefrontal cortex DARPP-32 mRNA in completed suicide victims with schizophrenia. Schizophr Res 103, 192-200, https://doi.org/10.1016/j.schres.2008.05.014 (2008).

31. Clinton, S. M. et al. Dopaminergic abnormalities in select thalamic nuclei in schizophrenia: involvement of the intracellular signal integrating proteins calcyon and spinophilin. Am J Psychiatry 162, 1859-1871, https://doi.org/10.1176/appi.ajp.162.10.1859 (2005).

32. Hakak, Y. et al. Genome-wide expression analysis reveals dysregulation of myelination-related genes in chronic schizophrenia. Proc Natl Acad Sci USA 98, 4746-4751, https://doi.org/10.1073/pnas.081071198 (2001). 
33. Wada, A. et al. Increased ratio of calcineurin immunoreactive neurons in the caudate nucleus of patients with schizophrenia. Prog Neuropsychopharmacol Biol Psychiatry 37, 8-14, https://doi.org/10.1016/j.pnpbp.2012.01.005 (2012).

34. Wada, A. et al. Prominent increased calcineurin immunoreactivity in the superior temporal gyrus in schizophrenia: A postmortem study. Psychiatry Res 247, 79-83, https://doi.org/10.1016/j.psychres.2016.11.018 (2017).

35. Greengard, P., Allen, P. B. \& Nairn, A. C. Beyond the dopamine receptor: the DARPP-32/protein phosphatase-1 cascade. Neuron 23, 435-447 (1999)

36. Gerber, D. J. et al. Evidence for association of schizophrenia with genetic variation in the 8p21.3 gene, PPP3CC, encoding the calcineurin gamma subunit. Proc Natl Acad Sci USA 100, 8993-8998, https://doi.org/10.1073/pnas.1432927100 (2003).

37. Horiuchi, Y. et al. Support for association of the PPP3CC gene with schizophrenia. Mol Psychiatry 12, 891-893, https://doi. org/10.1038/sj.mp.4002019 (2007).

38. Kinoshita, Y. et al. No association with the calcineurin A gamma subunit gene (PPP3CC) haplotype to Japanese schizophrenia. J Neural Transm (Vienna) 112, 1255-1262, https://doi.org/10.1007/s00702-004-0261-5 (2005).

39. Sanders, A. R. et al. No significant association of 14 candidate genes with schizophrenia in a large European ancestry sample: implications for psychiatric genetics. Am J Psychiatry 165, 497-506, https://doi.org/10.1176/appi.ajp.2007.07101573 (2008).

40. Xi, Z. et al. No association between PPP3CC and schizophrenia in the Chinese population. Schizophr Res 90, 357-359, https://doi. org/10.1016/j.schres.2006.10.012 (2007)

41. Mathieu, F. et al. Association between the PPP3CC gene, coding for the calcineurin gamma catalytic subunit, and bipolar disorder. Behav Brain Funct 4, 2, https://doi.org/10.1186/1744-9081-4-2 (2008).

42. Miyakawa, T. et al. Conditional calcineurin knockout mice exhibit multiple abnormal behaviors related to schizophrenia. Proc Natl Acad Sci USA 100, 8987-8992, https://doi.org/10.1073/pnas.1432926100 (2003).

43. Itokawa, M., Arinami, T., Futamura, N., Hamaguchi, H. \& Toru, M. A structural polymorphism of human dopamine D2 receptor, D2(Ser311->Cys). Biochem Biophys Res Commun 196, 1369-1375, https://doi.org/10.1006/bbrc.1993.2404 (1993).

44. Arinami, T. et al. Association of dopamine D2 receptor molecular variant with schizophrenia. Lancet 343, 703-704 (1994).

45. Glatt, S. J. \& Jonsson, E. G. The Cys allele of the DRD2 Ser311Cys polymorphism has a dominant effect on risk for schizophrenia: evidence from fixed- and random-effects meta-analyses. Am J Med Genet B Neuropsychiatr Genet 141B, 149-154, https://doi. org/10.1002/ajmg.b.30273 (2006)

46. He, H. et al. Associations between dopamine D2 receptor gene polymorphisms and schizophrenia risk: a PRISMA compliant metaanalysis. Neuropsychiatr Dis Treat 12, 3129-3144, https://doi.org/10.2147/NDT.S118614 (2016).

47. Gonzalez-Castro, T. B. et al. The role of C957T, TaqI and Ser311Cys polymorphisms of the DRD2 gene in schizophrenia: systematic review and meta-analysis. Behav Brain Funct 12, 29, https://doi.org/10.1186/s12993-016-0114-z (2016).

48. Itokawa, M. et al. Sequestration of the short and long isoforms of dopamine D2 receptors expressed in Chinese hamster ovary cells. Mol Pharmacol 49, 560-566 (1996).

49. Hausdorff, W. P., Caron, M. G. \& Lefkowitz, R. J. Turning off the signal: desensitization of beta-adrenergic receptor function. FASEB J4, 2881-2889 (1990)

\section{Acknowledgements}

This work was supported in part by the Strategic Research Program for Brain Sciences from AMED under Grant Numbers JP19dm0107086 (Y.K.), JP19dm0107107 (H.Y.), JP19dm0107104 (A.K.), by the Grant-in-Aid for Scientific Research on Innovative Areas from the MEXT under Grant Number JP16H06277 (H.Y.), and by the Grants-in-Aid from the Ministry of Education, Culture, Sports, Science and Technology of Japan under Grant Number 15K19739 (Y.K.). In addition, this study was supported by the Collaborative Research Project of Brain Research Institute, Niigata University under Grant Numbers 201917 (Y.K.). We thank Ms. C. Watanabe and Ms. H. Onuma for their contribution to coordinating donations, Mr. S. Yamamoto for his contribution to Choju Medical Institute Fukushimura Hospital brain collection. We also thank the families of the deceased for the donations of brain tissue and their time and effort devoted to the consent process and interviews.

\section{Author contributions}

Y.K. and M.H. designed the study. Y.K. and M.H. performed the experiments. Y.K., M.H., J.M., A.N., H.N., A.K., H.A., Y.H. and H.Y. collected postmortem brain samples and clinical information. Y.K. and M.H. undertook the statistical analysis. Y.K. wrote the first draft. All authors contributed to and have approved the final manuscript.

\section{Competing interests}

The authors declare no competing interests.

\section{Additional information}

Supplementary information is available for this paper at https://doi.org/10.1038/s41598-019-51456-7.

Correspondence and requests for materials should be addressed to Y.K.

Reprints and permissions information is available at www.nature.com/reprints.

Publisher's note Springer Nature remains neutral with regard to jurisdictional claims in published maps and institutional affiliations.

Open Access This article is licensed under a Creative Commons Attribution 4.0 International License, which permits use, sharing, adaptation, distribution and reproduction in any medium or format, as long as you give appropriate credit to the original author(s) and the source, provide a link to the Creative Commons license, and indicate if changes were made. The images or other third party material in this article are included in the article's Creative Commons license, unless indicated otherwise in a credit line to the material. If material is not included in the article's Creative Commons license and your intended use is not permitted by statutory regulation or exceeds the permitted use, you will need to obtain permission directly from the copyright holder. To view a copy of this license, visit http://creativecommons.org/licenses/by/4.0/.

(C) The Author(s) 2019 